\title{
Welcome Message from the Editors
}

\author{
Dear CSIAM-AM Reader:
}

On behalf of the editorial board and the China Society for Industrial and Applied Mathematics (CSIAM), it is our pleasure to welcome you to the first issue of the CSIAM Transactions on Applied Mathematics (CSIAM-AM). The goal of CSIAM-AM is to provide you, the valuable reader, with original research articles or reviews of high quality on a topic in applied mathematics that highlight the key role of mathematics in advancing modern science and technology.

The journal comes at the most exciting and critical time for applied mathematics as its relevance has never been so clear. The rapid developments of big data and artificially intelligence have greatly expanded the application scope of applied mathematics, and presented unprecedented opportunities and challenges to applied mathematics. CSIAMAM grows out of the strong demand of a quality platform for communicating significant research findings in applied mathematics that address interesting and challenging scientific problems. It will also be the flagship journal of CSIAM, a fast-growing society with over 10,000 individual non-student members.

The objective of CSIAM-AM is to publish only quality manuscripts and to maintain the quality as we move on. As an important step to achieve the objective, we are fortunate to have a distinguished group of mathematicians as our founding members of the editorial board. Since the reception of the first submission in February 2019, the initial response of the journal has been overwhelming. We hope the journal will continue to reach to the right audience and expand our reader group.

Finally, we would like to take the opportunity to acknowledge the help of many individuals over the course of launching the journal. In particular, the inauguration issue would not be possible without the generosity of the board members and reviewers who handled the first wave of submissions in a timely fashion, the staffs of the Global Science Press and CSIAM for their professional assistance, and members of the CSIAM Committee on Publications for their efforts.

Please enjoy the issue and we look forward to your future submissions.

Sincerely,

Pingwen Zhang, Editor-in-Chief and President of CSIAM

Gang Bao, Managing Editor

CSIAM Transactions on Applied Mathematics 